\title{
Bosonic gas as a Galactic Dark Matter Halo
}

\author{
L. Arturo Ureña-López ${ }^{1, *}$ and Argelia Bernal ${ }^{2}, \oplus$ \\ ${ }^{1}$ Departamento de Fúsica, División de Ciencias e Ingenierías, Campus León, \\ Universidad de Guanajuato, C.P. 37150, León, Guanajuato, México \\ ${ }^{2}$ Max-Planck-Institut für Gravitationsphysik (Albert-Einstein-Institut), Am Mühlenberg 1, 14476 Potsdam, Germany
}

(Dated: September 18, 2018)

\begin{abstract}
We study in detail the properties of gravitationally-bounded multi-state configurations, made of spin-zero bosons, in the Newtonian regime. We show that the properties of such configurations, in particular their stability, depend upon how the particles are distributed in the different states they are composed of. Numerical techniques are used to distinguish between stable and unstable solutions, and to determine the final configurations they evolve towards to. Multi-state equilibrium configurations can be used as models of galactic halos made of scalar field dark matter, whose rotation curves appear more realistic than in the case of single-state configurations.
\end{abstract}

PACS numbers: 95.35.+d,98.62.Gq,04.62.+v,04.40.-b

\section{INTRODUCTION}

It has been known for a long time that, within the context of Einstein's General Relativity, the luminous matter content of galaxies cannot explain the so-called Rotational Curves (RC) [1 3 ], which are still considered one of the cornerstone evidence for the existence of nonbaryonic dark matter. There are many candidates for dark matter particles, the most popular ones are known as weak interactive massive particles (WIMPS) [4 [6]. The accepted paradigm that describes the way in which those particles form structures is the so called Lambda Cold Dark Matter $(\Lambda \mathrm{CDM})$ model [7 9$]$.

An interesting alternative some of us have been working on is to consider a (real) scalar field as a dark matter candidate, a hypothesis that has been widely explored in the specialized literature by many other authors 10 25]; see also 26] for a comprehensive review. In most scalar field models, the dark matter particle is an ultralight massive boson, with a Compton wavelength of astrophysical proportions and a very large mean number density, so that their collective behavior is well described by a classical scalar field. The Scalar Field Dark Matter (SFDM) model, as we shall call it in general, offers the same results as the $\Lambda \mathrm{CDM}$ model at large scales, up to linear order perturbations [14, 16, 23, 25, 27, 28].

The RC problem has also been addressed using scalar fields, see for instance 10 12, 17, 29]). These works considered the dark matter halo as a Newtonian BoseEinstein Condensate (BEC), in which the scalar field dynamics is driven by the so-called Schroedinger-Poisson system of equations. However, none of the studies carried on so far have not shown, undeniably, that these scalar field models can account for all features of realistic galactic halo.

The modeling of scalar field halos was based on the (nodeless) ground state solutions of the SP system, which

\footnotetext{
* lurena@fisica.ugto.mx

† abernal@aei.mpg.de
}

is the only stable solution, and the predicted rotation curves are marginally in agreement with the observed ones 11 $13,30-32$. In all cases above, the only stable scalar field configuration is that in which boson particles are all in the ground state. The ground state is the only stable solution of the SP system against gravitational perturbations; other excited configurations are intrinsically unstable [18, 32] (see also 33, 34] and references therein).

The main purpose in this work is to further explore the proposal that was first put forward by Matos \& UreñaLópez in Ref. [35]: that realistic scalar field galaxy halos must be comprised of multi-state configurations. As we shall show, equilibrium configurations of the SP system can be constructed in which many-particle states coexist simultaneously, so that the whole system is stable under small (radial) perturbations 1 . Probably not surprisingly, we have found that RC could be better fitted by these many-particle systems.

The plan of the paper is as follows. In Sec. III we present the mathematical theory behind multi-particle states. In Sec. III we show that their general properties depend upon the distribution of the particles in the different excited states. In Sec. IV] we give numerical evidence that there are stable configurations under small radial perturbations, and investigate the late time behavior of unstable configurations. The RC curves predicted by stable multi-state configurations are calculated in Sec. VI. Finally, some conclusions are given in Sec. VII.

\section{MATHEMATICAL BACKGROUND}

Here we give a brief description of a gravitational bounded system of self-gravitating scalar field particles following the argumentation in the seminal paper [38], see

\footnotetext{
1 The relativistic version of the multi-state hypothesis was studied recently in [36, 37], in which stability was also confirmed.
} 
also 33, 34, 39 41. We pay special attention to the key aspects needed to build systems that have particles in the ground state but also in the excited states [35].

We start by assuming a spherically symmetric metric of the form

$$
d s^{2}=-\alpha^{2}(t, r) d t^{2}+a^{2}(t, r) d r^{2}+r^{2} d \Omega,
$$

in units of $\hbar=c=1$. The many boson-system is then described by a (secondly quantized) real scalar field operator of the form 42$]$

$$
\hat{\Phi}=\sum_{n l m}\left[\hat{b}_{n l m} \Phi_{n l m}(t, \mathbf{x})+\hat{b}_{n l m}^{\dagger} \Phi_{n l m}^{*}(t, \mathbf{x})\right],
$$

where $\hat{b}_{n l m}$ and $\hat{b}_{n l m}^{\dagger}$ are usual annihilation and creation quantum operators, which obey the commutation relations

$$
\begin{aligned}
{\left[\hat{b}_{n l m}, \hat{b}_{n^{\prime} l^{\prime} m^{\prime}}^{\dagger}\right] } & =\delta_{n n^{\prime}} \delta_{l l^{\prime}} \delta_{m m^{\prime}}, \\
{\left[\hat{b}_{n l m}, \hat{b}_{n l m}\right] } & =\left[\hat{b}_{n l m}^{\dagger}, \hat{b}_{n^{\prime} l^{\prime} m^{\prime}}^{\dagger}\right]=0 .
\end{aligned}
$$

The field coefficients $\Phi_{n l m}$ satisfy the Klein Gordon (KG) equation in a curved spacetime

$$
\left(\square-\mu^{2}\right) \Phi_{n l m}(t, \mathbf{x})=0,
$$

with $\square=(1 / \sqrt{-g}) \partial_{\mu}\left(\sqrt{-g} \partial^{\mu}\right)$ the covariant d'Alembertian operator, and $\mu$ is the mass of the bosons. The most general solution of Eq. (44) is of the form

$$
\Phi_{n l m}(t, \mathbf{x})=R_{n l}(t, r) Y_{l m}(\theta, \varphi),
$$

where we $R_{n l}(t, r)$ is the radial function to be determined from the KG equation. The scalar product of the functions above is defined as

$$
\left(\Phi_{n l m}, \Phi_{n^{\prime} l^{\prime} m^{\prime}}\right) \equiv-i \int_{\Sigma} \Phi_{n l m} \partial_{\mu} \Phi_{n^{\prime} l^{\prime} m^{\prime}}^{*} n^{\mu} \alpha \sqrt{\gamma} d \Sigma,
$$

where $\gamma$ is the determinant of the 3-dim metric on the spacelike hypersurface $\Sigma, n^{\mu}$ is a timelike unit vector orthogonal to $\Sigma$, and $d \Sigma$ is the volume element. In our case, see Eqs. (11) and (5), Eq. (6) reads

$$
\left(\Phi_{n l m}, \Phi_{n^{\prime} l^{\prime} m^{\prime}}\right)=-i \delta_{l l^{\prime}} \delta_{m m^{\prime}} \int_{V} R_{n l} \partial_{t} R_{n^{\prime} l^{\prime}}^{*} a^{2}(t, r) r^{2} d r
$$

where we have made use of the orthogonality condition of the spherical harmonics $Y_{l m}$.

Assuming that there exists a vacuum state defined by

$$
\hat{b}_{n l m}|0,0, \ldots, 0\rangle=0, \quad \forall(n, l, m)
$$

we can construct the orthonormal many-particle states

$$
\begin{aligned}
|Q\rangle & =\left|N_{100}, N_{200}, N_{21-1}, N_{210}, \ldots\right\rangle, \\
& \equiv \frac{\left(\hat{b}^{\dagger}\right)^{N_{100}}\left(\hat{b}^{\dagger}\right)^{N_{200}} \ldots}{N_{100} ! N_{200} ! \cdots}|0,0, \ldots 0\rangle,
\end{aligned}
$$

composed of many scalar particles distributed in sets of ${ }^{i} N_{n l m}$ particles of mass $\mu$ with angular momentum $l$ and azimuthal momentum $m$; the $n$ sub-index labels the eigenstates according to their radial function $R_{n l}$. Notice that many-particle states are constructed from the vacuum through the repeated application of the creation operators $\hat{b}^{\dagger}$.

On the other hand, the gravitational field is a classical field whose dynamics is described by the Einstein equations

$$
G_{\alpha \beta}=8 \pi G\left\langle Q\left|: \hat{T}_{\alpha \beta}:\right| Q\right\rangle,
$$

where the source on the r.h.s. is the expectation value of the energy-momentum tensor operator

$$
\hat{T}_{\alpha \beta}=\partial_{\alpha} \hat{\Phi} \partial_{\beta} \hat{\Phi}-\frac{1}{2} g_{\alpha \beta}\left(\partial^{\sigma} \hat{\Phi} \partial_{\sigma} \hat{\Phi}+\mu^{2} \hat{\Phi}^{2}\right) .
$$

Notice that we are implicitly assuming the so called normal ordering operation, $: \hat{T}_{\alpha \beta}$ :, so that

$$
\begin{array}{r}
: \hat{b}_{n l m} \hat{b}_{n l m}^{\dagger}:=\hat{b}_{n l m}^{\dagger} \hat{b}_{n l m}, \\
\left\langle 0, \ldots, 0,0\left|: \hat{T}_{\alpha \beta}:\right| 0,0, \ldots, 0\right\rangle=0,
\end{array}
$$

and then the (divergent) vacuum energy density identically vanishes.

The orthogonality of the quantum states ensures that the expectation value is given as a superposition of the expectation values of the energy-momentum tensor components for each individual state, that is,

$$
\left\langle Q\left|: \hat{T}_{\alpha \beta}:\right| Q\right\rangle=\sum_{n=1}^{\infty} \sum_{l=1}^{n-1} \sum_{m=-l}^{l}\left\langle N_{n l m}\left|: \hat{T}_{\alpha \beta}:\right| N_{n l m}\right\rangle,
$$

where we are defining the single states

$$
\left|N_{n l m}\right\rangle \equiv\left|0,0, \ldots, N_{n l m}, 0, \ldots, 0\right\rangle .
$$

Hence, the Einstein equations (10) read

$$
G_{\alpha \beta}=8 \pi G \sum_{n, l, m} T_{\alpha \beta(n l m)},
$$

where

$$
\begin{aligned}
T_{\alpha \beta(n l m)}= & \partial_{\alpha} \Phi_{n l m} \partial_{\beta} \Phi_{n l m}^{*}-\frac{1}{2} g_{\alpha \beta}\left(\partial^{\sigma} \Phi_{n l m} \partial_{\sigma} \Phi_{n l m}^{*}\right. \\
& \left.+\mu^{2} \Phi_{n l m} \Phi_{n l m}^{*}\right),
\end{aligned}
$$

and we also normalized the eigenfunctions so that $\Phi \rightarrow$ $\Phi / \sqrt{2 N_{n l m}}$.

Therefore, in the case when particles populate various excited levels, the source of the Einstein equations (15) is equivalent to the energy momentum tensor of many (independent) classical complex scalar fields $\Phi_{n l m}(t, \mathbf{x})$ minimally coupled to gravity. Each one of such scalar fields accounts for only one of the excited single states (14), and its dynamics is given by its own KG equation (44) 38, 42]. 
Finally, we consider the Newtonian limit of the coupled Einstein-Klein-Gordon (EKG) equations (4) and (15), which results in the so-called Schrödinger-Poisson (SP) system[43]

$$
\begin{aligned}
\nabla^{2} U & =\sum_{n l m}\left|\Psi_{n l m}\right|^{2}, \\
i \partial_{t} \Psi_{n l m} & =-\frac{1}{2} \nabla^{2} \Psi_{n l m}+U \Psi_{n l m},
\end{aligned}
$$

where $\Psi_{n l m}$ is related to $\Phi_{n l m}$ by

$$
\sqrt{8 \pi G} \Phi_{n l m}(t, \mathbf{x})=e^{-i \mu t} \Psi_{n l m}(t, \mathbf{x}) .
$$

Then, the Newtonian version of the EKG equations describes the dynamics of non-relativistic wave functions which are coupled among themselves through the Newtonian gravitational potential $U$.

Once in the non-relativistic regime, we can define physical quantities like the kinetic $K$ and gravitational $W$ energies, and the total number of particles $\mathcal{N}$. These quantities can be explicitly given in terms of the Newtonian fields as 32

$$
\begin{aligned}
K & =-\frac{1}{2} \sum_{n, l, m} \int\left(\Psi_{n l m}^{*} \nabla^{2} \Psi_{n l m}+\Psi_{n l m} \nabla^{2} \Psi_{n l m}^{*}\right) d \\
W & =\sum_{n, l, m} \int U\left|\Psi_{n l m}\right|^{2} d v \\
\mathcal{N} & =\sum_{n, l, m} \int\left|\Psi_{n l m}\right|^{2} d v
\end{aligned}
$$

These expressions will be useful later to monitor the numerical evolution of multi-state configurations studied in Sec. IV.

\section{MIXED NEWTONIAN STATES}

In this section we construct solutions of the SP system (17) when $\mathcal{N}$ bosons are allowed to occupy $\mathcal{I}$ different levels, all of which, for simplicity in the discussion, will have zero angular momentum $(l=0, m=0)$. Hence, the states are of the form $|Q\rangle=\left|N_{1}, N_{2}, N_{3}, \ldots, N_{\mathcal{I}}\right\rangle$. Assuming spherical symmetry, we then have

$$
\begin{aligned}
\frac{1}{r^{2}} \frac{\partial^{2}\left(r^{2} U\right)}{\partial r^{2}} & =\sum_{n=1}^{\mathcal{I}}\left|\Psi_{n}\right|^{2}, \\
i \frac{\partial \Psi_{n}}{\partial t} & =-\frac{1}{2 r^{2}} \frac{\partial^{2}\left(r^{2} \Psi_{n}\right)}{\partial r^{2}}+U \Psi_{n}, \quad n=1, . .
\end{aligned}
$$

First of all, we look for stationary equilibrium configurations in the form

$$
\Psi_{n}=e^{-i \gamma_{n} t} \phi_{n}(r)
$$

for which the system (20) becomes

$$
\begin{aligned}
\frac{1}{r^{2}} \frac{d^{2}\left(r^{2} U\right)}{d r^{2}} & =\sum_{n=1}^{\mathcal{I}}\left|\phi_{n}\right|^{2}, \\
\frac{1}{r^{2}} \frac{d^{2}\left(r^{2} \phi_{n}\right)}{d r^{2}} & =2\left(U-\gamma_{n}\right) \phi_{n}, \quad n=1, . ., \mathcal{I}
\end{aligned}
$$
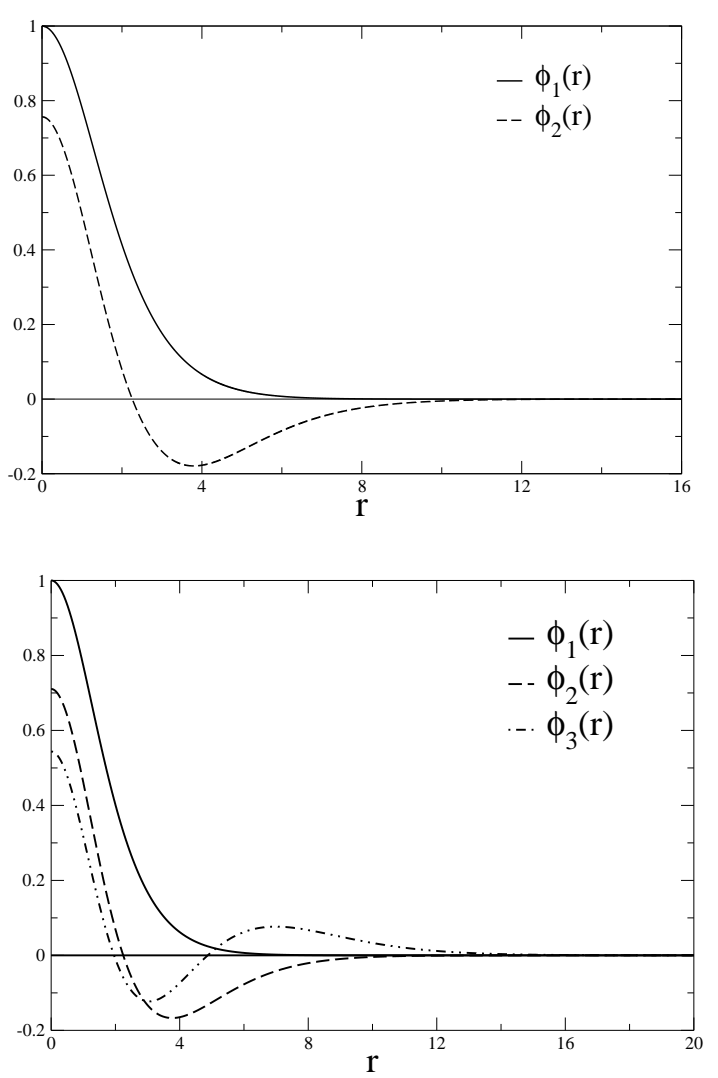

FIG. 1. Radial functions $\phi_{1}$ and $\phi_{2}$ for the mixed configuration $\left|N_{1}, 1.1\right\rangle$, and $\phi_{1}, \phi_{2}$ and $\phi_{3}$ for the mixed configuration $\left|N_{1}, 0.96,0.91\right\rangle$ see also the first and third entries in Table $\square$ and text below for details about its construction.

Eqs. (22) will be solved under the following boundary conditions. In order to obtain regular solutions at the origin, we demand that the spatial derivatives of all $\phi_{n}$ and $U$ must be zero at this point, but we arbitrarily prescribe the central values of all $\phi_{n}$, as these values are the free parameters of the solutions. Also, we impose $\phi_{n}(r) \rightarrow 0$ and $U(r)=-\mathcal{N} / r$ as $r \rightarrow \infty$ because we are looking for bounded configurations.

With these boundary conditions the system becomes an eigenvalue problem. Given the central values $\left\{\phi_{n}(0)\right\}$ there are unique values $\left\{\gamma_{n}\right\}$ and $U(0)$ for which the boundary conditions are satisfied. The numerical solutions are then found by using a shooting method to integrate Eqs. (22) from $r=0$ up to the numerical boundary $r=r_{\max }$, with $\left\{\gamma_{n}\right\}$ and $U(0)$ playing the role of shooting parameters.

Because the equations are integrated in a finite numerical domain, it is more convenient to introduce more detailed specifications to the boundary conditions at $r \rightarrow \infty$. We use the asymptotic behavior of the solutions: the gravitation potential goes like $U(r \rightarrow \infty)=$ $-\mathcal{N} / r$, and the radial functions behave as $\phi_{n}(r \rightarrow \infty) \sim$ 


\begin{tabular}{|c|c|c|c|c|c|}
\hline State & $\phi_{n}(0)$ & $\gamma_{n}$ & $K$ & $W$ & $\mathcal{N}$ \\
\hline$\left|N_{1}, 1.1\right\rangle$ & $\phi_{2}(0)=0.756$ & $\gamma_{1}=-1.033, \gamma_{2}=-0.574$ & 1.846 & -3.691 & 3.493 \\
\hline$\left|N_{1}, 1.6\right\rangle$ & $\phi_{2}(0)=0.934$ & $\gamma_{1}=-1.163, \gamma_{2}=-0.677$ & 2.272 & -4.544 & 3.945 \\
\hline$\left|N_{1}, 0.96,0.91\right\rangle$ & $\phi_{2}(0)=0.710, \phi_{3}(0)=0.543$ & $\gamma_{1}=-1.185, \gamma_{2}=-0.712, \gamma_{3}=-0.471$ & 2.407 & -4.819 & 4.520 \\
\hline
\end{tabular}

TABLE I. Central values of excited states $\phi_{n}(0)$, eigenvalues $\gamma_{n}$, kinetic $K$ and gravitational $W$ energies, and the total number of particles $\mathcal{N}$ of three mixed states, all of them with $\phi_{1}(0)=1.0$. The labeling of the mixed states is in terms of the $\eta$ parameters defined in Eq. (25). See text bellow for details.

$\exp \left(-\sqrt{-2 \gamma_{n}} r\right)$. Then, more suitable boundary conditions are

$$
\begin{array}{r}
U\left(r_{\max }\right)+r_{\max } U_{\max }^{\prime}=0, \\
\phi_{n}^{\prime}\left(r_{\max }\right)+\sqrt{2 \gamma_{n}^{2}} \phi_{n}\left(r_{\max }\right)=0 .
\end{array}
$$

The shooting procedure is then used for different values of $r_{\max }$. As $r_{\max }$ is increased the shooting parameters converge, and we choose as solutions those which satisfy the boundary conditions (23) within a prescribed tolerance.

Before we show the numerical results we make some remarks about notation. In the case when all the particles are in the one same level, the system (22) consists of only one Schrödinger equation and only one wave function in the source term of the Poisson equation. Such system has been widely studied in 32], where several solutions $\left\{\phi_{n}, \gamma_{n}, U\right\}$ have been calculated.

The main difference between the functions $\phi_{n}$ is that they have $(n-1)$-nodes in their radial profile. The state corresponding to the zero-node function $\phi_{1}$ has the lowest (negative) total energy $E=K+W$, the lowest energy eigenvalue $\gamma_{1}$, and is correspondingly the less massive; this state is called the ground state. The other solutions with nodes are more massive and have larger energy values than the ground state; they are called excited states.

In this spirit, we shall call mixed states to those configurations in which single states $\phi_{n}$ are present simultaneously. For instance, the simplest mixed state configuration consists of the ground state $\phi_{1}$ and the first excited state $\phi_{2}$, and then it is characterized by quantum state $\left|N_{1}, N_{2}\right\rangle$. In the next section we construct some of these configurations.

\section{A. Ground-first mixed states $\left|N_{1}, N_{2}\right\rangle$}

Let us start with the simplest mixed state, that composed of the ground state $\phi_{1}$ plus the first excited state $\phi_{2}$. At this point, it is not necessary to solve Eqs. (22) for each possible pair $\left(\phi_{1}(0), \phi_{2}(0)\right)$ in order to find the complete space of solutions.

Instead, it is worth to use the scaling symmetry that the complete SP system obeys [32]; for the particular case here, it reads

$$
\begin{aligned}
\left\{\phi_{1}, \phi_{2},\right. & \left.\gamma_{1}, \gamma_{2}, U, r\right\} \rightarrow \\
& \left\{\lambda^{2} \hat{\phi}_{1}, \lambda^{2} \hat{\phi}_{2}, \lambda^{2} \hat{\gamma}_{1}, \lambda^{2} \hat{\gamma}_{2}, \lambda^{2} \hat{U}, \lambda^{-1} r\right\},
\end{aligned}
$$

where $\lambda$ is an arbitrary parameter.

This means that once we have found a solution to the SP system for given values of $\left(\hat{\phi}_{1}(0), \hat{\phi}_{2}(0)\right)$, there is a complete set solutions each of which are related to each other just by the scaling transformation (24). This set we will call it a family of solutions. Different families are then found by taking different central values of $\left(\hat{\phi}_{1}(0), \hat{\phi}_{2}(0)\right)$.

The central values $\phi_{1}(0), \phi_{2}(0)$ of the solutions in a family, as a consequence of the scaling relation (24), will be located along the straight line defined by the origin and the given point $\left(\hat{\phi}_{1}(0), \hat{\phi}_{2}(0)\right)$ on the plane $\phi_{1}, \phi_{2}$. Therefore, once all the solutions with the same value $\hat{\phi}_{1}(0)$ and different $\hat{\phi}_{2}(0)$ are known, and their respective families have been calculated, the complete space of solutions can be constructed as the collection of all families of solutions. For simplicity in the notation, we will drop the caret symbol from the field quantities, in the understanding that we are dealing with scaled quantities.

Useful quantities to characterize mixed states are the ratios of the number of particles in different states with respect to the ground state; we define these ratios as

$$
\eta_{n} \equiv \mathcal{N}_{n} / \mathcal{N}_{1}
$$

where by definition $\eta_{1}=1$, and the total number of particles is $\mathcal{N}=\mathcal{N}_{1}\left(1+\eta_{2}+\eta_{3}+\cdots\right)$. Notice that $\eta_{n}$ are invariant quantities under the scaling relationship (24).

We calculated solutions with $\phi_{1}(0)=1$ for different values of $\phi_{2}(0)$, and we observed that $\eta_{2}$ increases monotonically as $\phi_{2}(0)$ grows, starting at the value $\eta_{2}=0$ when $\phi_{2}(0)=0$ (no particles in the excited state, $N_{2}=$ $0)$. This behavior of $\eta_{2}$ allows us to choose it as a free parameter instead of $\phi_{2}(0)$, and then $\left(\phi_{1}(0), \eta_{2}\right)$ will be the representative parameters in the construction of the solutions. This is a convenient option because, as we will see in the next section, there is evidence that the stability under radial perturbations of a ground-first state depends mainly on the values of $\eta_{2}$.

In order to fix the value of $\eta_{2}$, it is necessary to add the differential expressions for the number of particles $\mathcal{N}_{j}$

$$
\frac{d \mathcal{N}_{n}}{d r}=\phi_{j}^{2} r^{2}, \quad n=1, . ., \mathcal{I},
$$

in the system (22). The boundary conditions for these equations are given at the origin $N_{n}(0)=0$, and the desired value of $\phi_{2}$ is imposed at $r_{\max }$. We then solve 
the system of equations (22) and (26) using a shooting method; this time, however, the shooting parameters are $\eta_{2}(0), \gamma_{1}, \gamma_{2}$ and $U(0)$.

Once again, the complete space of configurations is formed by the collection of families of solutions, each one characterized by the same value of $\hat{\phi}_{1}(0)$ and different $\eta_{2}$. As a consequence of (24), the physical quantities of the solutions for each family will be related by

$$
\begin{aligned}
\left\{N_{1}, N_{2}, K, W\right\} & \rightarrow \\
& \left\{\lambda \hat{N}_{1}, \lambda \hat{N}_{2}, \lambda^{3} \hat{K}, \lambda^{3} \hat{W}\right\} .
\end{aligned}
$$

In the top panel of fig. 1 we show typical radial functions of a (mixed) ground-first state, the zero-node radial function corresponds to the ground state whereas the one-node radial function corresponds to the first excited state. The mixed state was constructed with $\phi_{1}(0)=1.0$ and $\eta_{2}=1.1$, and so we labeled it as $\left|\mathcal{N}_{1}, 1.1\right\rangle$.

In the first row of Table I. we show the scalar field central value for the excited state $\phi_{2}(0)$, the frequencies for both states, $\gamma_{1}$ and $\gamma_{2}$, as well as the kinetic and the gravitational energies, and the total number of particles of the system. The same quantities are shown in the second row of the same table for the mixed state $\left|\mathcal{N}_{1}, 1.6\right\rangle$, which was constructed with $\phi_{1}(0)=1.0$ and $\eta_{2}=1.6$.

Following a similar procedure to the explained above we also constructed systems where particles are coexisting in the ground state and in the first and second excited states. In the bottom panel of Fig. 1, we show the radial functions for one of those systems with $\phi_{1}(0)=1.0$, and $\eta_{2}=\eta_{3}=1.0 ;$ we called it $\left|\mathcal{N}_{1}, 1.0,1.0\right\rangle$.

In Table \ we show its energy eigenvalues $\gamma_{1,2,3}$ and other physical quantities. We were able to verify that, within the limitations imposed by the numerical error, all configurations we could find satisfy the virialization condition $2 K+W=0$.

We constructed several ground-first states for different values of $\phi_{1}(0)$ and $\eta_{2}$. In Fig. 3, we plot the total energy of each system in terms of the number of particles in the ground state and first excited states, $N_{1}, N_{2}$. It is possible to notice that the total energy for each system is negative, which implies that they are gravitationally bounded objects.

In Fig. 4, the energy eigenfrequencies $\gamma_{1}$ and $\gamma_{2}$ are shown; for all configurations $\gamma_{1}<\gamma_{2}$. Finally, in Fig. 5 . the kinetic energy of each state, $K_{1}$ and $K_{2}$, is shown. In contrast with the behavior of the eigenfrequencies, there are configurations for which $K_{1}<K_{2}$ if $\eta_{2}>1$, whereas $K_{1}>K_{2}$ if $\eta_{2}<1$.

\section{B. Mixed states as a generalization of single states}

We want to show now that mixed states are generalizations of single states. As mentioned before, if the number of particles $\mathcal{N}$ in an single state is fixed, whatever the ground or any of the excited states, the total energy $E$ takes a fixed value because of the eigenvalue

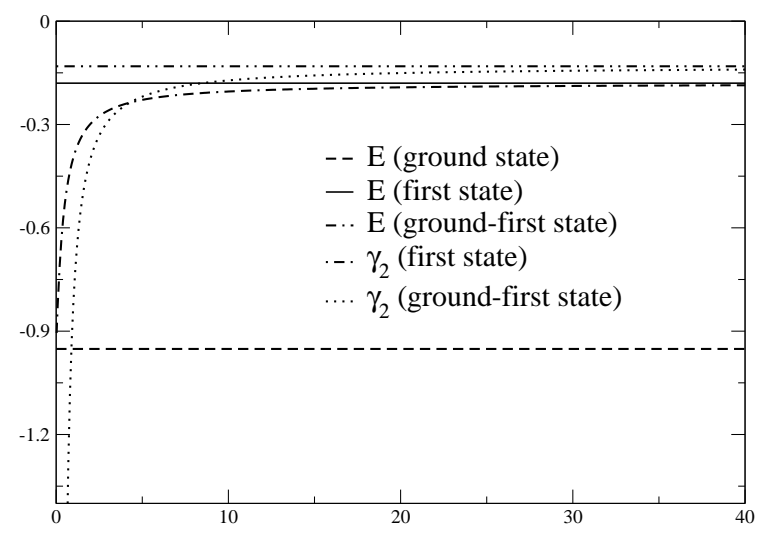

FIG. 2. Comparison of the total energy $E=K+W$ of a ground-first configuration with a fixed number of particles $\mathcal{N}=\mathcal{N}_{1}+\mathcal{N}_{2}=2.0622$, with two single-state configurations with the same number of total particles: one ground singlestate, and one first excited single-state. The total energy depends upon the fraction $\eta_{2}=\mathcal{N}_{2} / \mathcal{N}_{1}$, see Eq. (25). If $\mathcal{N}_{1} \gg \mathcal{N}_{2}, E$ goes to the energy value of the ground singlestate; if $\mathcal{N}_{1} \ll \mathcal{N}_{2}$, then $E$ goes to the energy value of the first excited single-state. We make a similar comparison with $\gamma_{2}$. If $\mathcal{N}_{1} \gg \mathcal{N}_{2}, \gamma_{2}$ takes very large negative values, whereas if $\mathcal{N}_{1} \ll \mathcal{N}_{2}$, then $\gamma_{2}$ approaches the value corresponding to a first excited single-state.

problem; this value however increases for larger number of nodes in the radial profile.

This behavior of $E$ changes radically if the same number of particles $\mathcal{N}$ are distributed in a mixed configuration. Now, the total energy of the system $E$ can take values from a continuum interval depending on how the particles populate the states of the mixed state.

In particular, for a ground-first configuration the total energy is a monotonically increasing function of $\eta_{2}$. If $\eta \sim 0, E \rightarrow E_{1}^{\text {(single) }}$, where $E_{1}$ is the total energy of a single ground state composed of $\mathcal{N}$ particles. On the other hand, if the particles are moved from the ground to the first excited state, the total energy of the mixed state takes continuum values, and $E \rightarrow E_{2}^{\text {(single) }}$, where $E_{2}^{(\text {single) }}$ is the total energy of a single first state, as the ground state is depopulated (in other words, $\eta_{2} \rightarrow \infty$ ).

In Fig. 2 we show this behavior of the total energy for a system of with a fixed total number of particles $\mathcal{N}=2.0622$. Notice that the values are within the range $E_{1}^{\text {(single })}<E<E_{2}^{\text {(single) }}$. The same kind of behavior was found for all other characteristic quantities, like the eigenfrequencies $\gamma_{n}$, see also Fig. 2.

From this perspective, we can say that, given a system of $\mathcal{N}$ particles in a single-state configuration, it is possible to change the properties of the quantities attached to it by moving particles away to populate other excited 


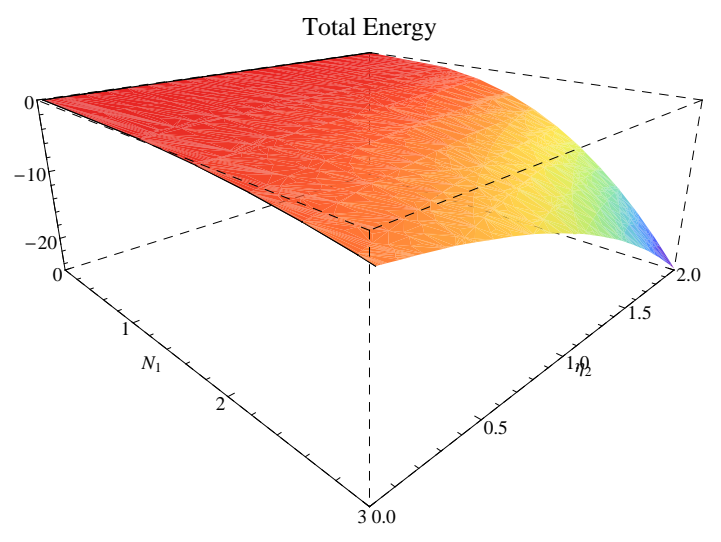

FIG. 3. Total energy of ground-first configurations in terms of $\mathcal{N}_{1}$ and $\mathcal{N}_{2}$. All of them are negative, which implies that the systems are gravitationally bounded.

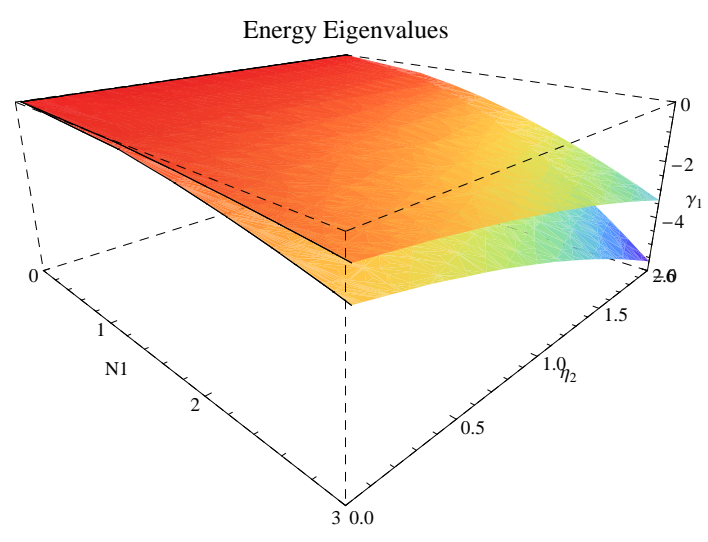

FIG. 4. Frequency eigenvalues $\gamma_{1}$ and $\gamma_{2}$ of ground-first configurations in terms of $\mathcal{N}_{1}$ and $\mathcal{N}_{2}$. For all the configurations it is satisfied that $\left|\gamma_{2}\right|<\left|\gamma_{1}\right|$.

states.

\section{STABILITY OF MIXED STATES UNDER SMALL PERTURBATIONS}

In order to promote the existence of mixed states beyond the mathematical context, it is first necessary to prove their stability. It is known that when all the particles are in the ground or in an excited state, the configuration is stable under small radial perturbations that strictly conserve the number of particles, $\delta N=0[17,[32]$.

Instead, if the system is considered open, so that $\delta N=$ 0 is not demanded, an excited state emits particles, loses its nodes, and eventually settles down on to a ground state 30, 32]. On the contrary, ground states can tolerate perturbations for which $\delta N \neq 0[44]$, and it is in this sense that ground states are said to be stable, whereas excited states are unstable.

It is then expected that the excited state of a groundfirst configuration should remain unstable if there are very few particles in the ground state. However, the re-

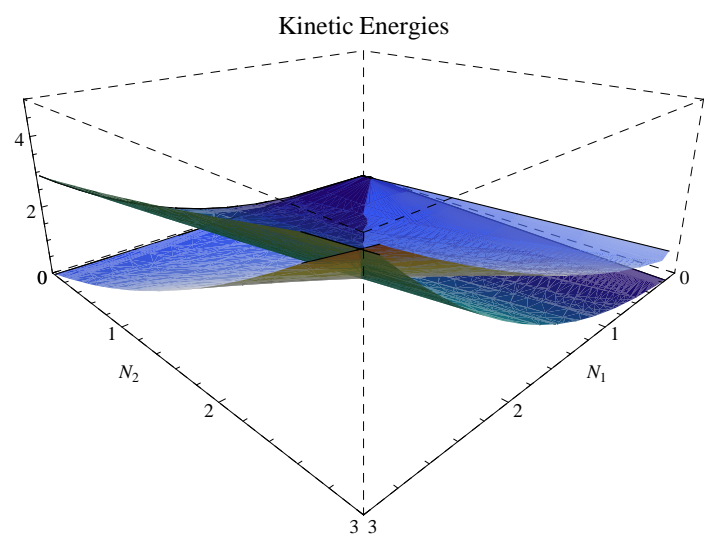

FIG. 5. Kinetic energy for each separate state $K_{1}, K_{2}$, in a ground-first configuration in terms of $\mathcal{N}_{1}$ and $\mathcal{N}_{2}$. The systems with $K_{1}>K_{2}$ correspond to $\eta_{2}<1\left(\mathcal{N}_{1}>\mathcal{N}_{2}\right)$, whereas those with $K_{1}<K_{2}$ correspond to $\eta_{2}>1\left(\mathcal{N}_{1}<\mathcal{N}_{2}\right)$.

sults in the previous section show that the properties of excited states change depending on how many particles populate the ground state.

This is actually the case of stability. We found that, by adding particles to the ground state, it is possible to construct ground-first configurations for which, under open conditions, the mixed state is stable under radial perturbations. As particles do not interact directly one with each other, the change in the stability of the excited state is produced just by the gravitational interaction with the ground state.

Stability studies of single configurations have been done with perturbation theory and full numerical evolutions. In principle, the stability of mixed states can be also studied perturbatively (a work that is worth a separate manuscript). Instead, we have chosen to evolve numerically mixed states not only to determine their stability, but also their late time behavior. We have done this for several ground-first configurations with different values of $\eta_{2}$.

\section{A. Numerical perturbation}

The evolution of the mixed states is done by solving the discretized version of the time dependent SP system (20), taking as the initial data the functions of the different unperturbed states $\Psi_{n}$, see Eq. (21), constructed in the previous section. We consider no perturbations other than those introduced by the finite differencing error in the numerical integration.

The procedure followed in the construction of the solutions to Eqs. (20) can be summarized as follows.

1. The numerical grid is populated with the initial data

$$
\Psi_{n}^{N}\left(t_{0}=0, k \Delta r\right)=\phi_{n}(k \Delta r)
$$


where $t_{0}$ is an arbitrary initial time that we choose to be zero, $\Delta r$ is the spatial resolution of the grid, the super-index $N$ labels the discretized numerical solution, $k$ is an integer that labels the grid and $n$ runs from $1 \ldots \mathcal{I}, \mathcal{I}$ the number of populated states.

2. The gravitational potential $U^{N}(0, k \Delta r)$ is obtained by introducing $\Psi_{n}^{N}$ in Eq. (20).

3. Using the obtained gravitational potential $U^{N}$ in Eqs. (20), each populated state of the system is leaped forward in time a step $\Delta t$ getting $\Psi_{n}^{N}(\Delta t, k \Delta r)$.

4. Repeating $j-1$ times the steps 2 and 3 above we obtain $\Psi_{n}^{N}(j \Delta t, k \Delta r)$ and $U^{N}((j-1) \Delta t, k \Delta r)$.

Because the SP system is discretized in order to solve it numerically, it is expected that the numerical solution $\Psi_{n}^{N}$ differs from the equilibrium value $\Psi_{n}$ in Eq. (21) by a numerical error $\Delta \Psi_{n}$, i.e.,

$$
\Psi_{n}^{N}(j \Delta t, k \Delta r)=e^{-i \gamma_{n} j \Delta t} \phi_{n}(k \Delta r)+\Delta \Psi_{n}(j \Delta t, k \Delta r) .
$$

We then say that the system is perturbed without considering an explicit perturbation, because the numerical error $\Delta \Psi_{n}$, that comes from the discretization, is considered as the perturbation itself; in fact, $\Psi_{n}^{N}$ behaves like a perturbed $\Psi_{n}$ during the evolution.

We have to stress that during the numerical evolution we allow the system to eject particles. With the implemented open boundary conditions in the code, the condition for which the number of particles has to be preserved is not maintained.

\section{B. Perturbing the ground state}

In Ref. 32 the discretization error was used to perturb a single ground state in order to study its stability and it was shown that the numerical evolution reproduces the results obtained with perturbation theory. Here we give a brief account of this result.

Using perturbation theory to first order, when a single ground state function $\Psi_{1}=\phi_{1}(r) e^{-i \gamma_{1} t}$ is perturbed with a small radial perturbation $\delta \Psi_{1}$, keeping the number of particles constant, a new oscillation mode $\sigma$ appears in the perturbed system $\Psi_{\text {pert }}=\Psi_{1}+\delta \Psi_{1}$. It is found that $\delta \Psi_{1}$ is regular, spatially localized, and has an harmonic time dependence (such time-dependence involves not only the new oscillation mode $\sigma$, but also the eigenvalue $\gamma_{1}$ ). The system is then said to be stable under small radial perturbations.

A quantity that gives relevant information about the new oscillation mode is the perturbed density. The ground state non-perturbed density $\rho_{1}=\left|\Psi_{1}\right|^{2}$ is time independent, whereas the perturbed one $\rho_{\text {pert }}=\left|\Psi_{\text {pert }}\right|^{2}$ has an harmonic time dependence and oscillates with an angular frequency $2 \pi \sigma$. To first order in $\delta \Psi_{1}$, the perturbed density is

$$
\rho_{\text {pert }}=\left|\Psi_{1}+\delta \Psi\right|^{2}=\rho_{1}(r)+\delta \rho(r) \cos (2 \pi \sigma t) .
$$

These perturbed ground state properties are also found when the ground state is evolved numerically without considering any explicit perturbation, except for that inherent to the discretization. Using as initial data the time independent function $\phi_{1}$ of a ground state, its temporal behavior is obtained as explained in the previous subsection. A quantity that is monitored throughout the evolution is the function $\operatorname{Re}\left[\Psi_{1}^{N}(0, t)\right]$, which behaves harmonically in time as expected. Its Fourier Transform (FT) shows a main harmonic mode that matches the eigenfrequency $\gamma_{1}$ of the unperturbed ground state.

The density $\rho_{1}^{N}(t, 0)=\left|\Psi_{1}^{N}(t, 0)\right|^{2}$ also shows an harmonic behavior in agreement with the perturbative result (29). It oscillates around the value of the nonperturbed density $\rho_{1}$, and from its FT we observe that its main oscillation mode coincides with $\sigma$. Finally, even with the open boundary conditions in the numerical code, the number of particles does not change, which is consistent with the results of perturbation theory.

\section{Perturbing stable mixed states}

In order to study the stability of a mixed state, we evolve it following the steps described in IVA The general idea is to compare the behavior of $\operatorname{Re}\left[\Psi_{n}^{N}(t, 0)\right]$, $\rho_{n}^{N}(t, 0)$, and the number of particles $\mathcal{N}_{n}^{N}(t)$ for each occupied state with the behavior of the corresponding quantities for a single ground state. We can conclude that there is evidence of the stability of the mixed configuration if those behaviors are similar.

In fig. 6] we show the numerical evolution of $\operatorname{Re}\left[\Psi_{n}^{N}(t, 0)\right]$ for a ground-first configuration with $\eta_{2}=$ 1.1 , i.e., a $\left|\mathcal{N}_{1}, 1.1\right\rangle$ state. The wave functions behave harmonically, and their FTs, presented in Fig. 7 show that the main harmonic mode of each $\Psi_{n}^{N}$ corresponds to the angular frequency $\gamma_{n}$ of the unperturbed $\Psi_{n}$.

Furthermore, in Fig. 8 we show the numerical values of the central densities $\rho_{n}^{N}=\left|\Psi_{n}^{N}(t, 0)\right|^{2}$, and it is clear that they oscillate closely around the value of the unperturbed densities (which are formally time-independent), $\rho_{1}=$ $\left|\phi_{1}\right|^{2}=1.0$ and $\rho_{2}=\left|\phi_{2}\right|^{2}=0.572$.

The bounded oscillations suggest that the numerical perturbations of each $\Psi_{n}$ are spatially localized and have an harmonic time dependence. In the bottom panel of Fig. 8, we show the central value of the total density $\rho^{N}=\rho_{1}^{N}+\rho_{2}^{N}$, with its corresponding FT in Fig. 9. As in the case of a single ground state, for which its quasinormal mode is the mean harmonic mode of the perturbed density, we expect that the mean harmonic mode shown in this figure corresponds to the characteristic oscillation mode of the perturbed state $\left|\mathcal{N}_{1}, 1.1\right\rangle$.

In Fig. 10, we verify that the number of particles for each occupied state is conserved separately, so that the 

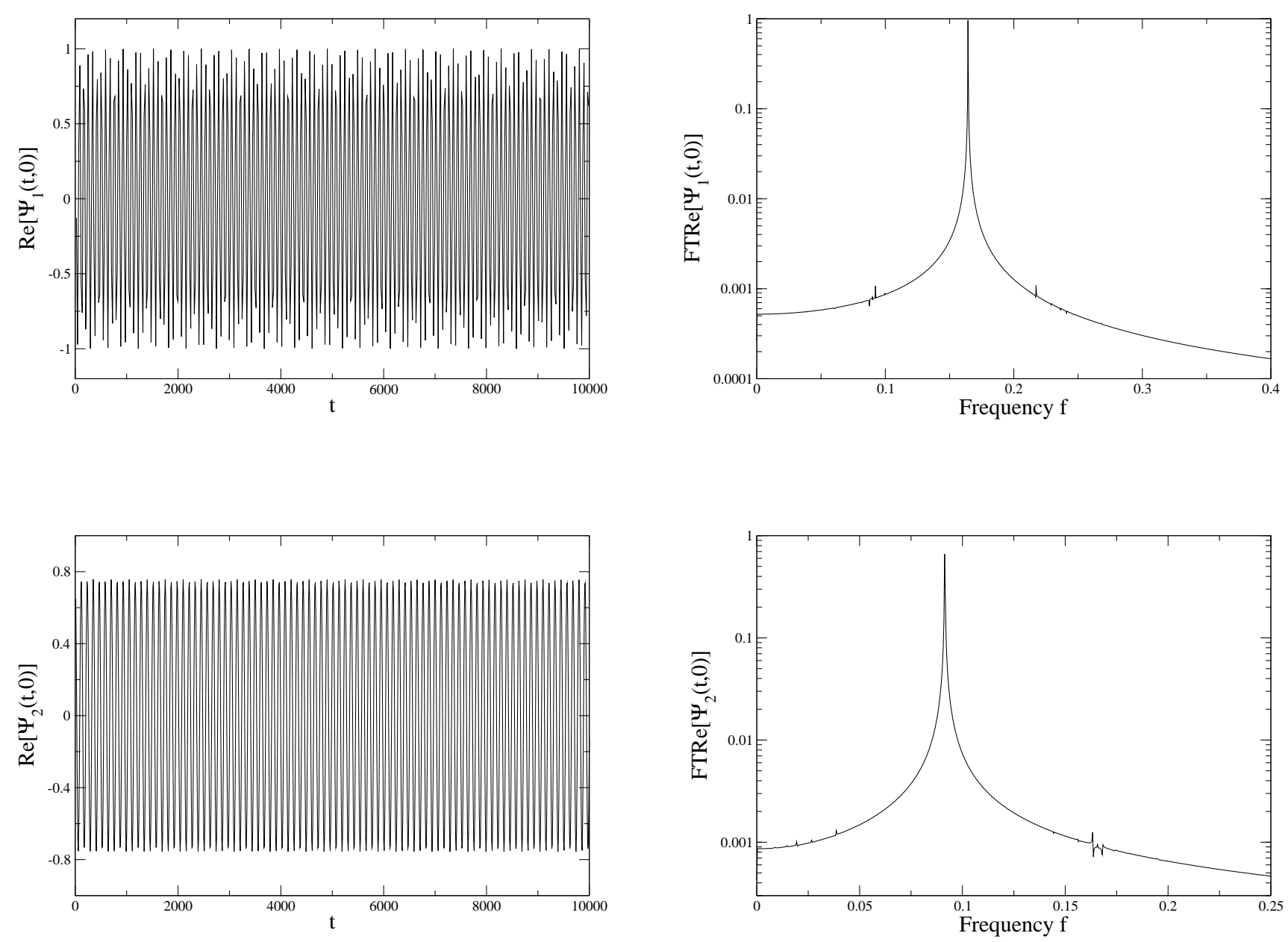

FIG. 6. Evolution of $\operatorname{Re}\left[\Psi_{1}^{N}(t, 0)\right]$ (top) and $\operatorname{Re}\left[\Psi_{2}^{N}(t, 0)\right]$ (bottom) for the mixed state $\left|N_{1}, \eta_{2}\right\rangle$. An harmonic behavior is observed, and the oscillation modes can be read from the corresponding FFT shown in Fig. 7

same happens for the total number of particles. In the bottom panel of Fig. 10, the relation $2 K+W$ for the system is presented, which in turn shows that the configuration remains virialized during the evolution.

\section{Perturbing unstable mixed states}

We performed the evolution for several ground-first states with $\phi_{1}(0)=1.0$ and different values of $\eta_{2}$. A general result is that if $\eta_{2}<1.1$ the mixed state behaves similarly as the state $\left|N_{1}, 1.1\right\rangle$. That is, each state of the configuration evolves harmonically with the main angular frequency $\gamma_{n}$ of the unperturbed wave function $\Psi_{n}$, the oscillations of the central densities $\rho_{n}^{N}$ are bounded, and the number of particles in each state is conserved. We take for granted that these characteristics are evidence

FIG. 7. (Top) FFT of $\operatorname{Re}\left[\Psi_{1}^{N}(t, 0)\right]$ for the $\left|N_{1}, \eta_{2}\right\rangle$ mixed state. The main mode at $f=0.164$ corresponds to the angular frequency $2 \pi f=1.033$ which coincides, in good approximation, with the value of $\gamma_{1}$ of the unperturbed wave function $\Psi_{1}$, see Table【 (Bottom) FFT of $\operatorname{Re}\left[\Psi_{2}^{N}(t, 0)\right]$ for the $\left|N_{1}, \eta_{2}\right\rangle$ mixed state; the main mode at $f=0.091$ corresponds to the angular frequency $2 \pi f=0.574$, which also coincides with the value of $\gamma_{2}$ of the unperturbed wave function $\Psi_{2}$, see Table 1

of the stability of these ground-first configurations.

On the other hand, the evolution of configurations with $\eta_{2}>1.1$ differ considerably. In the top panel of Fig. 12] we show the early evolution of the central densities $\rho_{n}^{N}$ for a ground-first configuration with $\phi_{1}(0)=1.0$ and $\eta_{2}=1.6$, which is the state $\left|N_{1}, 1.6\right\rangle$.

At the beginning, they just oscillate around the values of the unperturbed initial state, $\rho_{1}(0)=1.0$ and $\rho_{2}(0)=0.873$. However, after a while the amplitudes of the oscillations start to grow. In fact there is a period of time in which it is possible to fit each $\rho_{n}^{N}$ with a function of the form

$$
\rho_{n} \simeq a_{1} e^{a_{2} t} \cos \left(a_{3} t+a_{4}\right),
$$



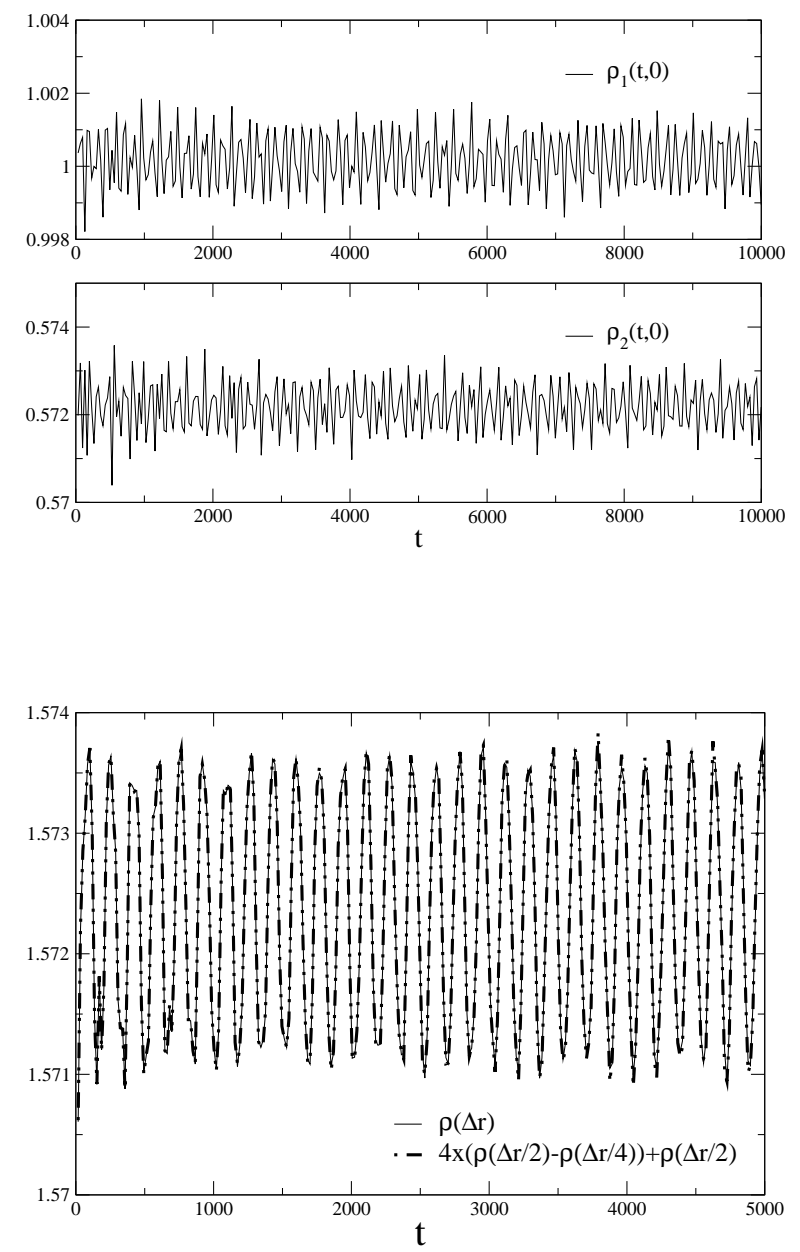

FIG. 8. (Top) The perturbed central densities $\rho_{n}^{N}=$ $\left|\Psi_{n}^{N}(t, 0)\right|^{2}$ for the $\left|N_{1}, \eta_{2}\right\rangle$ configuration are shown. They oscillate around the constant density values of the unperturbed states, corresponding to $\rho_{1}=1.0$ and $\rho_{2}=0.485$. Such oscillatory behavior is the expected one for stable states (see Sec. IVB for details). (Bottom) Overlap between $\rho^{N}(\Delta r)$ and $4\left(\rho^{N}(\Delta r / 2)-\rho^{N}(\Delta r / 4)\right)+\rho^{N}(\Delta r / 2)$. This plot shows that the numerical evolution is second order convergent.

where the $a$ 's are constants. This behavior suggest that the perturbations of the states $\Psi_{n}$, besides their harmonic time dependence, are exponentially unstable since $a_{2}>0$ in general.

From Fig. 12, it is possible to infer that the exponential growth of the density amplitude of the excited state, which starts almost from the very beginning of the evolution, acts as a trigger for the instability of the complete configuration. For completeness, we show in the top panel of Fig. 11 the convergence of $\rho_{12}^{N}(t, 0)$ for different spatial resolutions. We can conclude that the exponential growth of perturbations is a physical characteristic and not a spurious numerical result.

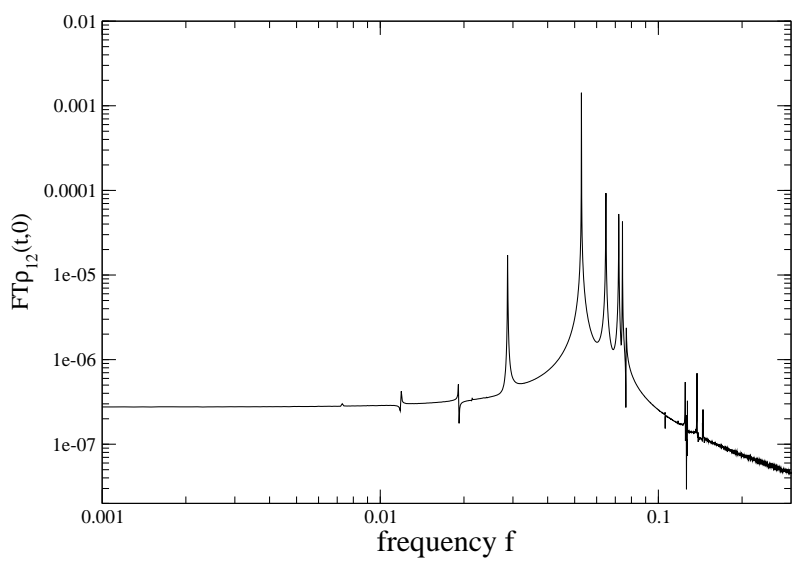

FIG. 9. FFT of the numerically-perturbed total density $\rho^{N}(t, 0)$. The main mode appears at $f=0.065$, which should correspond to the quasinormal mode of oscillation of the perturbed configuration.

Moreover, the number of particles in each state of the configuration $\left|N_{1}, 1.6\right\rangle$ is not conserved. In Fig. 14 we show that both the ground and the first excited states lose particles. Even more, the behavior of each $\operatorname{Re}\left[\Psi_{n}^{N}(t, 0)\right]$ also shows that their angular frequency change with time, and that only at the early stages of the evolution the frequencies coincide with the unperturbed values $\gamma_{1}=-1.163$ and $\gamma_{2}=-0.677$, see the top panel of Fig. 13 (the way in which the plotted quantities are computed is explained in the next section). Therefore, the configuration $\left|N_{1}, 1.6\right\rangle$ is not stable.

We performed the evolution for several configurations with different values of $\eta_{2}>1.2$, and we noticed that the speed of growth of the perturbation amplitudes increases for larger values of $\eta_{2}$. In order to quantify the speed of growth in terms of $\eta_{2}$, during the early times of the evolution we fit the central density of the excited state $\rho_{2}^{N}$ with a function of the form (30).

The values of coefficient $a_{2}$ for the exponential coefficient in terms of $\eta_{2}$ are plotted in Fig. 10. A linear extrapolation indicates that $a_{2} \rightarrow 0$ as $\eta_{2} \rightarrow 1.13$. As we already found that configurations with $\eta_{2}<1.1$ do not exhibit exponential growth, then we take $\eta_{2}^{\text {tresh }} \sim 1.13$ as a threshold value that separates stable and unstable configurations.

This result has been obtained from configurations with $\phi_{1}(0)=1.0$ and different values of $\eta_{2}$. Since mixed configurations obey the scaling symmetry (24), for which $\eta_{2}$ is an invariant quantity, then this threshold value should hold for all configurations. 

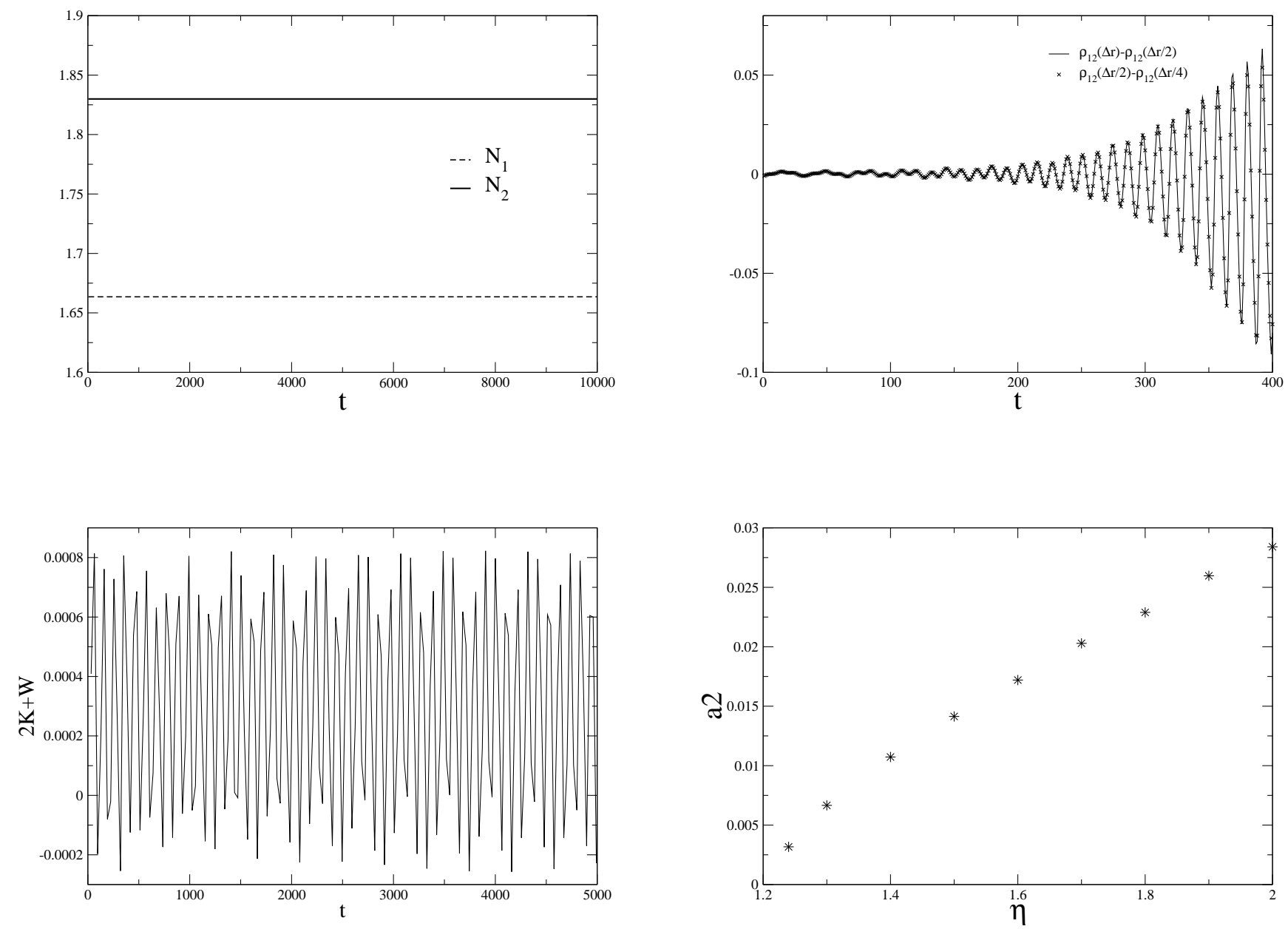

FIG. 10. (Left) Number of particles for each state of the mixed configuration $\left|N_{1}, \eta_{2}\right\rangle$ is shown. It is observed that the number of particles is conserved all along the numerical evolution. (Right) The value $2 K+W$ is shown. Since it oscillates around zero, it is inferred that the mixed configuration is a virialized system

\section{LATE TIME BEHAVIOR OF UNSTABLE MIXED STATES}

In this section we present evidence that shows that unstable ground-first configurations, when perturbed, evolve towards a stable configuration, in a process that parallels the evolution of single unstable states into the ground one, see [30].

During the stabilization process, we will see that the excited state loses particles and the node in its radial profile disappears. Likewise, the ground state loses particles as well, but a node appears in its radial profile. In any case, it will be possible to identify the final stable configuration the system is settle down onto.

A useful procedure is to follow the evolution of the

FIG. 11. (Top) The perturbed central density $\rho_{2}^{N}$ of a unstable configuration with $\eta=1.6$ is shown. The overlap between $\rho_{2}^{N}(\Delta r)-\rho_{2}^{N}(\Delta r / 4)$ and $4\left(\rho_{2}^{N}(\Delta r / 2)-\rho_{2}^{N}(\Delta r / 4)\right)$ show that $\rho_{2}^{N}$ converges at second order. (Bottom) Coefficient $a_{2}$ of the exponential function (30) used to fit the growth in the oscillations of the $\rho_{2}^{N}$ for different unstable configurations are shown. It is null for the threshold value of $\eta_{2} \simeq 1.13$, see text for details.

effective eigenfrequency defined by

$\gamma_{i}^{N} \equiv \frac{1}{\int\left|\Psi_{i}^{N}\right|^{2} d v}\left(-\frac{1}{2} \int \Psi_{i}^{N} \nabla^{2} \Psi_{i}^{N} d v+\int U\left|\Psi_{i}^{N}\right|^{2} d v\right)$,

which is a generalization of the eigenfrequency obtained from the Schroedinger equation (20) in the case of stationary solutions of the form (21), i.e.,

$$
\gamma_{i}=\frac{1}{\int \phi_{n}^{2} d v}\left(-\frac{1}{2} \int \phi_{i} \nabla^{2} \phi_{i} d v+\int U \mid \phi_{i}^{2} d v\right) .
$$

The precise identification of the final configuration is a tricky task, as the ground and the excited state inter- 
change the node in their radial profile during the evolution. We have found that the labeling of the states, whether ground or first excited state, depends basically in the relative values of $\gamma_{1}^{N}$ and $\gamma_{2}^{N}$. As in the case of stationary solutions, we shall call ground state the state with the lowest value of $\gamma^{N}$.

Now, we are going to describe the main stages in the evolution of the ground-first configuration $\left|N_{1}, 1.6\right\rangle$ in terms of $\rho_{i}^{N}(t, 0), \gamma_{i}^{N}(t)$, and $\mathcal{N}_{i}^{N}(t)$. At the beginning of the evolution, $\rho_{i}^{N}(t, 0)$ oscillate with small amplitude around the unperturbed values $\rho_{1}=1.0$ and $\rho_{2}=0.873$, see the top panel in Fig. (12). The same behavior is found for $\gamma_{i}^{N}(t)$, which oscillate around $\gamma_{1}=-1.163$ and $\gamma_{2}=-0.677$, see the top panel in Fig. 13 .

During this stage $\gamma_{1}^{N}<\gamma_{2}^{N}$, we can clearly see that the radial profile of $\operatorname{Re}\left[\Psi_{1}^{N}(t, 0)\right]$ shows no nodes, whereas $\operatorname{Re}\left[\Psi_{1}^{N}(t, 0)\right]$ has one node. The number of particles $\mathcal{N}_{i}^{N}$ in each state remains constant, see Fig. 14 .

Later on, the amplitude of each $\rho_{i}^{N}(t, 0)$ starts to grow exponentially as discussed before, see for instance Fig. 12 Meanwhile, $\gamma_{i}^{N}$ oscillate with bigger amplitudes and move away from their initial values, in such a way that $\gamma_{2}^{N}$ decreases and $\gamma_{1}^{N}$ increases, see Fig. (13). At the same time, the number of nodes in the radial profiles $\operatorname{Re}\left[\Psi_{i}^{N}(t, 0)\right]$ changes quickly and the number of particles of both states starts to decrease, see Fig. (14).

Then the system enters in a stage in which it experiences the most violent changes. The amplitudes of the oscillations of $\rho_{i}^{N}(t, 0)$ and $\gamma_{i}^{N}(t)$ becomes larger (Figs. (12) and (13) ), and the values of $\gamma_{i}^{N}(t)$ intersect and continue moving away from each other. The number of nodes in the radial profiles continues changing, and the number of particles of each state decreases quickly, see Fig. (14).

Finally, the system relaxes and the values of $\rho_{i}^{N}(t, 0)$ and $\gamma_{i}^{N}(t)$ start to converge and oscillate around a fixed value. It can be noticed that $\gamma_{1}^{N}>\gamma_{2}^{N}$, and that the radial profiles have interchanged nodes. Also the number of particles in each state stabilizes around fixed values. All this description strongly suggests that the system is reaching a stable configuration.

In order to verify that the final configuration really corresponds to a stable one, we read from the numerical results a set of parameters that can help us to construct an equilibrium configuration as described in Sec. III A. For the particular case studied here, we find that $\rho_{2}^{N}=$ 0.617 (recall that the excited state became the ground one), and $\eta_{2}=\mathcal{N}_{1}^{N} / \mathcal{N}_{2}^{N}=0.544$.

We then construct an equilibrium configuration with the aforementioned values as input parameters, compute all relevant quantities, and compare them with their (numerically) evolved counterparts. The resulting values are shown and compared in Table [I] Based on the fact that those values coincide in good approximation, we can affirm that the unstable configuration $\left|N_{1}, 1.6\right\rangle$, evolves towards a stable configuration with $\eta_{2}=0.544$ and $\phi_{1}(0) \approx 1.557$.

Long evolutions for several configurations for which initially $\eta_{2}>1.1$ and $\phi_{1}(0)=1.0$, show that their behavior is very much like the one observed in the unstable configuration with $\eta=1.6$. In all cases, they evolve towards a stable configuration, as exemplified in Fig. (15).

Here we have defined another scale-invariant quantity for the ratio between the eigenfrequencies of the equilibrium configurations,

$$
\Gamma_{n} \equiv \gamma_{n} / \gamma_{1}
$$

such that again $\Gamma_{1}=1$. We can plot the resulting values of ground-first stationary solutions on the plane $\left(\eta_{2}, \Gamma_{2}\right)$, which are then represented by the solid line in Fig. 15. We notice that equilibrium configurations with initial $\eta_{2}>1.1$ evolve towards an equilibrium configuration with $\eta_{2}<1.1$.
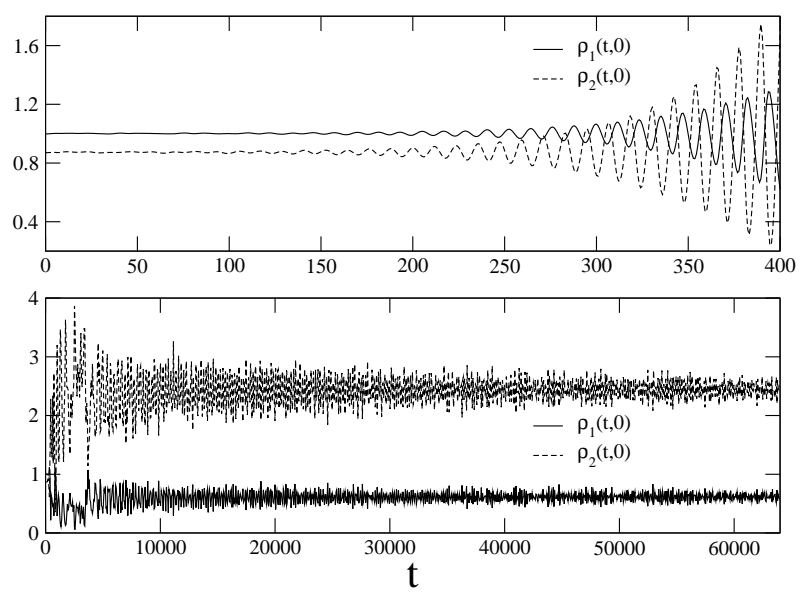

FIG. 12. Evolution of $\rho_{i}^{N}(t, 0)$ for a unstable ground-first configuration with $\eta=1.6$. (Top) Early time behavior; (Bottom) late time behavior. The system eventually settles down onto a stable, stationary, configuration.

\section{ROTATION CURVES IN SCALAR FIELD GALAXY HALOS}

We mentioned before that our main motivation for studying mixed configurations was the possibility that a scalar field would be the dark matter in galaxies. For that, we wanted to explore the capabilities of a scalar field to form realistic galaxy halos.

A crude estimation of rotation curves in mixed states was first presented in Ref. 35], under the (then untested) assumption that they were stable, a feature we can now consider firmly confirmed by the results of the present work.

For the mixed states studied in the previous sections, we can calculate the velocity of test particles moving along circular orbits in the gravitational potential 


\begin{tabular}{|c|c|c|c|c|c|}
\hline & $\phi_{1}(0)$ & $\phi_{2}(0)$ & $\gamma_{1}$ & $\gamma_{2}$ & $U(0)$ \\
\hline Final & 1.563 & 0.786 & -1.355 & -0.690 & -2.461 \\
\hline
\end{tabular}

TABLE II. Central values of the excited states $\phi_{n}(0)$, eigenvalues $\gamma_{n}$, kinetic $K$ and gravitational $W$ energies, and the total number of particles $\mathcal{N}$ of the mixed states. Because of the similarity between the final values of the evolved configuration shown in Figs. 12, 15, it can be concluded that the final state certainly corresponds to an equilibrium configuration.
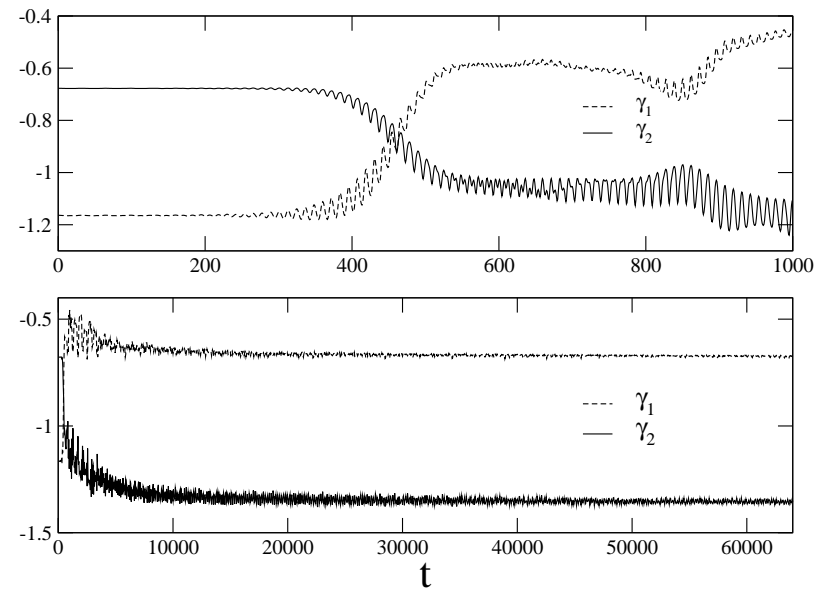

FIG. 13. Evolution of $\gamma_{i}^{N}$ for a unstable ground-first configura tion with $\eta=1.6$. (Top) Early time behavior; (Bottom) late time behavior, see text below.

sourced by the mixed states configurations via the Newtonian formula

$$
v(r)=\sqrt{\mathcal{N}(t, r) / r},
$$

where $\mathcal{N}(t, r)$ is the total number of particles inside the radius $r$ obtained from the numerical equilibrium configurations of the SP system (20).

The results are shown in Fig. 16. We can see a noticeable improvement in the flatness of the rotation curve at large radii as long as more excited states are taken into account. Some comments are in turn.

The circular velocity of mixed configurations shows some flat profile at intermediate radii, whereas the typical Keplerian tail shows up at large radii, indicating that at the end we are dealing with a localized object of finite size. We also note the existence of some ripples in the velocity profile, which are a consequence of the nodes present in the radial functions of excited states $\phi_{n}$.

The height and position of the first peak in the velocity profile are approximately set by the ground-state wave function $\phi_{1}$, whereas the total size of the objects is fixed by the profile of the most excited state.

At this point, we cannot say that mixed states are already strong candidates to explain galaxy halos, but for

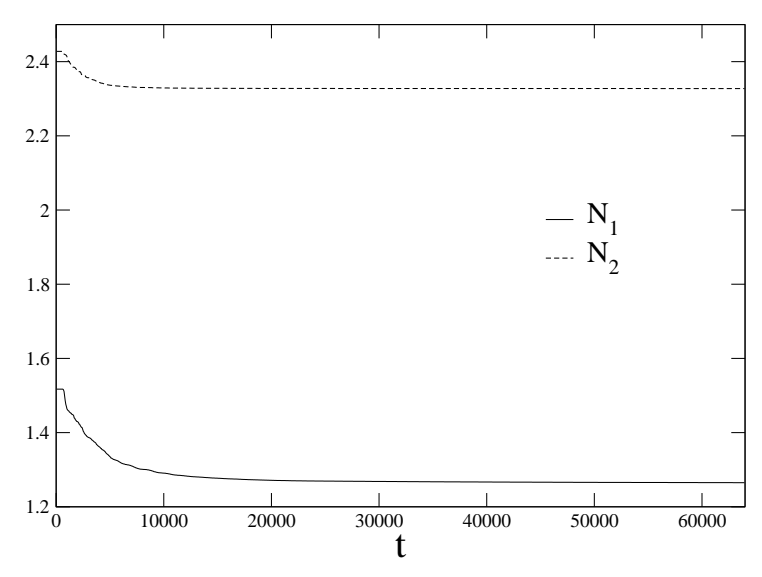

FIG. 14. Evolution of $N_{1}$ and $N_{2}$ for a unstable ground-first configuration with $\eta=1.6$.

that a more complete study would be necessary, like the one pursued in $10-13]$, where also baryons were included in the equations of motion.

We can though provide some clues about the possible physical features of a scalar field galaxy halo. In most scalar field dark matter models, the scalar field mass is usually very light, around $\mu \sim 10^{-23} \mathrm{eV}$; for such a small value, it was possible to consider that single state configuration, whether the ground or any of the excited ones, accounted for the complete halo configuration. This was a central assumption in most of previous references about scalar field dark matter models [10, 12, 16, 21, 27].

The Compton wavelength of the scalar field is very large for usual standards, because $\lambda_{C}=\mu^{-1} \simeq 10 \mathrm{pc}$. In the case of a Newtonian configuration, the size of the bounded object scales like $R=r / \lambda^{2}$, where $\lambda$ is the scaling parameter in Eq. (24), which in turn is related to the central field value of the configuration through $\lambda^{2}=\phi_{1}(0)$.

Following previous works, the scaling parameter is estimated to be $\lambda \sim 10^{3}[32,45]$. This implies that a stable single-state equilibrium configuration can model a galaxy halo of a size of around $5-7 \mathrm{kpc}$. However, larger galaxy halos, as is the typical case, are out of the capabilities of single state configurations. This limitation can be noticed, for instance, in the fits done in [13]. 


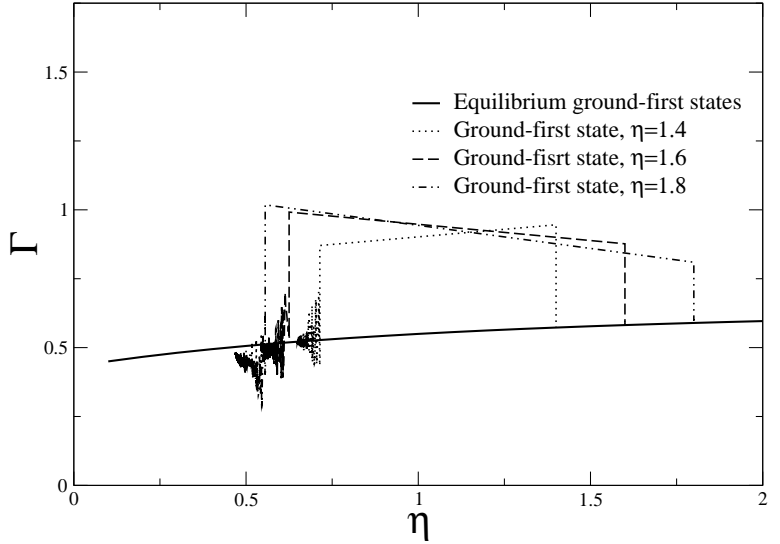

FIG. 15. Fate of unstable configurations with $\eta=1.4, \eta=1.6$ and $\eta=1.8$, in terms of parameter $\Gamma$, see Eq. (33). Notice that the configuration oscillates around the line representing stationary equilibrium configurations on the stable branch $\eta_{2}<1.13$, see Sec. IVD.

Mixed states can alleviate this limitation. As the size of the configuration is determined by the most excited state, the scalar field halos can be as large as necessary, and this helps to fit better the RC in real halos.

Moreover, as already noticed in Ref.[35], mixed states provides us with more free parameters to play with. The extra parameters are the occupation numbers of the mixed state, namely $N_{1}, N_{2}, N_{3}$, etc. Except for the limitations imposed by stability, these values would be determined initially, before the collapse of the scalar field configuration, by the local environment a scalar halo could be subjected to during the cosmological evolution.

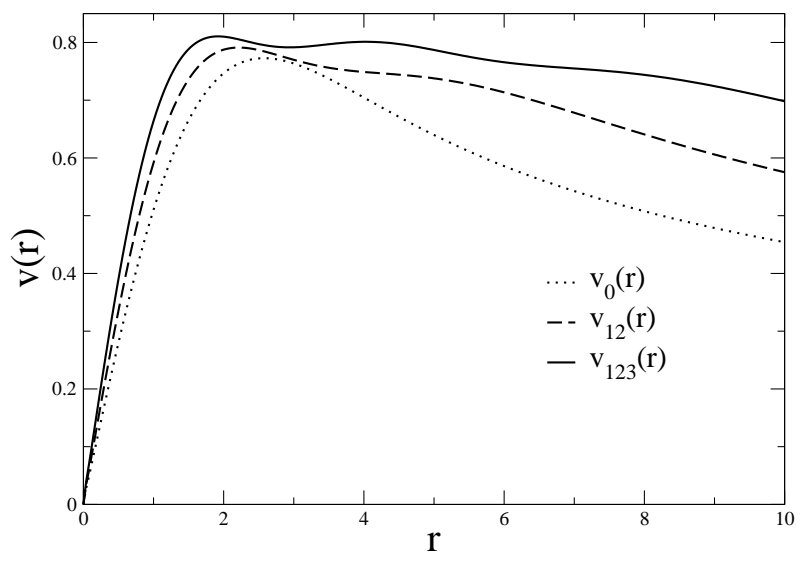

FIG. 16. The rotation curve $v(r)$, see Eq. (34), for the single ground state $\left|N_{1}=2.0622\right\rangle$, and the mixed configurations $\left|N_{1}, 1.1\right\rangle$ and $\left|N_{1}, 0.96,0.91\right\rangle$, see also Table【 Notice that the flatness of the curve is improved if more excited states are taken into account.

\section{CONCLUSIONS}

We have shown, for the first time, the existence of stable many-particle states made of scalar field in the Newtonian regime; such states are the generalization of the well-known boson stars that have been exhaustively studied in the literature. The possibility of mixed states was already suggested in the seminal paper of Ruffini \& Bonazolla about boson stars, but their existence and properties had not been studied before

Detailed instructions were provided for their construction and classification, but more importantly is that we established simple and sufficient criteria to determine their stability properties. Even though Newtonian configurations obey a scaling relationship, it was possible to define invariant parameters that allows us to follow their evolution and determine their final fate.

Some remarks were given regarding the importance these results may have for scalar field dark matter models in describing the properties of galaxy halos. However, more work is needed to have a complete picture of all possibilities offered by scalar fields and their gravitationally bounded configurations. This is an objective we will pursue in future work that we expect to report soon elsewhere.

\section{ACKNOWLEDGMENTS}

We are grateful to Jose Socorro for his encouragement and support. We also thank Juan Barranco, Ulises Nucamendi, and Olivier Sarbach for helpful discussions, and Francisco S. Guzmán for sharing his numerical code, which we modified to account for multi-state configurations. This work was partially supported by CONACYT grant 56946, DAIP and PROMEP-UGTO-CA-3. AB acknowledges support from CONACYT grant 47641, and the kind hospitality of the Departamento de Física of the Universidad de Guanajuato, for a postdoctoral stay during which this work was initiated.
[1] V. C. Rubin, Science, 220, 1339 (1983).

[2] M. Persic, P. Salucci, and F. Stel, Mon. Not. Roy. Astron. Soc., 281, 27 (1996), arXiv:astro-ph/9506004
[3] J. R. Primack, (2001), arXiv:astro-ph/0112255.

[4] S. Colafrancesco, AIP Conf. Proc., 1206, 5 (2010), arXiv:1004.3869 [astro-ph.CO] 
[5] A. Del Popolo, Astron. Rep., 51, 169 (2007) arXiv:0801.1091 [astro-ph]

[6] M. Taoso, G. Bertone, and A. Masiero, JCAP, 0803. 022 (2008), arXiv:0711.4996 [astro-ph].

[7] K. A. Olive, (2010), arXiv:1005.3955 [hep-ph].

[8] A. J. Benson, (2010), arXiv:1006.5394 [astro-ph.CO]

[9] D. Larson et al. (2010), arXiv:1001.4635 astro-ph.CO]

[10] S.-J. Sin, Phys. Rev., D50, 3650 (1994), arXiv:hep-ph/9205208.

[11] S. U. Ji and S. J. Sin, Phys. Rev., D50, 3655 (1994), arXiv:hep-ph/9409267.

[12] J. Lesgourgues, A. Arbey, and P. Salati, New Astron. Rev., 46, 791 (2002)

[13] A. Arbev, J. Lesgourgues, and P. Salati, Phys. Rev., D68, 023511 (2003), arXiv:astro-ph/0301533

[14] T. Matos, J. A. Vazquez, and J. Magana, (2008), arXiv:0806.0683 [astro-ph]

[15] T. Matos and L. A. Urena-Lopez, Class. Quant. Grav., 17, L75 (2000), arXiv:astro-ph/0004332.

[16] T. Matos and L. A. Urena-Lopez, Phys. Rev., D63. 063506 (2001), arXiv:astro-ph/0006024.

[17] J.-w. Lee and I.-g. Koh, Phys. Rev., D53, 2236 (1996), arXiv:hep-ph/9507385

[18] J.-W. Lee, (2008), arXiv:0801.1442 [astro-ph].

[19] J.-W. Lee, Phys. Lett., $\quad$ B681, 118 (2009), arXiv:0805.2877 [astro-ph]

[20] J.-W. Lee, S. Lim, and D. Choi, (2008),

[21] W. Hu, R. Barkana. and A. Gruzinov, Phvs. Rev. Lett. 85, 1158 (2000), arXiv:astro-ph/0003365.

[22] W. Hu, Astrophys. J., 506,485 (1998), arXiv:astro-ph/9801234.

[23] T.-P. Woo and T. Chiueh, Astrophys. J., 697, 850 (2009), arXiv:0806.0232 [astro-ph]

[24] L. A. Urena-Lopez, (2008), arXiv:0806.3093 [gr-qc]

[25] A. P. Lundgren, M. Bondarescu, R. Bondarescu, and J. Balakrishna, Astrophys. J., 715, L35 (2010), arXiv:1001.0051 [astro-ph.CO].
[26] T. Matos and L. A. Urena-Lopez, Int. J. Mod. Phys., D13, 2287 (2004), arXiv:astro-ph/0406194.

[27] V. Sahni and L.-M. Wang, Phys. Rev., D62, 103517 (2000), arXiv:astro-ph/9910097

[28] T. Matos, J.-R. Luevano, I. Quiros, L. A. Urena-Lopez, and J. A. Vazquez, Phys. Rev., D80, 123521 (2009), arXiv:0906.0396 [astro-ph.CO]

[29] F. E. Schunck, (1998), arXiv:astro-ph/9802258.

[30] F. S. Guzman and L. A. Urena-Lopez, Astrophys. J., 645, 814 (2006), arXiv:astro-ph/0603613

[31] T. D. Lee and Y. Pang, Nucl. Phys., B315, 477 (1989).

[32] F. S. Guzman and L. A. Urena-Lopez, Phys. Rev., D69, 124033 (2004), arXiv:gr-qc/0404014

[33] F. E. Schunck and E. W. Mielke, Class. Quant. Grav., 20, R301 (2003), arXiv:0801.0307 [astro-ph].

[34] P. Jetzer, Phys. Rept., 220, 163 (1992).

[35] T. Matos and L. A. Urena-Lopez, Gen. Rel. Grav., 39, 1279 (2007).

[36] A. Bernal, J. Barranco, D. Alic, and C. Palenzuela, Phys. Rev., D81, 044031 (2010), arXiv:0908.2435 [gr-qc],

37 A. Bernal, J. Barranco, D. Alic, and C. Palenzuela, AIP Conf. Proc., 1241, 335 (2010).

\begin{tabular}{|l|l|l|l|}
38 & R. Ruffini and S. Bonazzola, & Phys. Rev., 187, 1767 \\
\hline
\end{tabular} (1969).

39] E. Seidel and W.-M. Suen, Phys. Rev., D42, 384 (1990)

[40] J. Balakrishna, E. Seidel, and W.-M. Suen, Phys. Rev., D58, 104004 (1998), arXiv:gr-qc/9712064.

[41] J. Balakrishna, R. Bondarescu, G. Daues, F. Siddhartha Guzman, and E. Seidel, Class. Quant. Grav., 23, 2631 (2006), arXiv:gr-qc/0602078.

[42] N. D. Birrell and P. C. W. Davies, Cambridge, Uk: Univ. Pr. ( 1982) 340p.

[43] R. Friedberg, T. D. Lee, and Y. Pang, Phys. Rev. D, 35, 3640 (1987).

[44] A. Bernal and F. S. Guzman, Phys. Rev., D74, 063504 (2006), arXiv:astro-ph/0608523.

[45] F. S. Guzman and L. A. Urena-Lopez, Phys. Rev., D68, 024023 (2003), arXiv:astro-ph/0303440 